\title{
UK INFANTILE SPASMS STUDY: EFFECT OF TIME TO TREATMENT AND AGE AT ONSET ON DEVELOPMENTAL OUTCOME
}

The effects of lead time to treatment (time from onset of spasms to start of treatment), age at onset of spasms, etiology, and treatment on developmental outcome at 4 years were investigated using multiple linear regression in 77 infants with spasms treated in the UK Infantile Spasms Study (UKISS). Age of onset ranged from $<1$ to 10 months (mean 5.2), and lead time to treatment was 7 days or less in 11, 8-14 days in 16, 15 days to 1 month in 8,1-2 months in $15,>2$ months in 21 and not known in 6 . The Vineland Adaptive Behavior Scales (VABS) scores showed a 3.1 decrease with each month of reduction in age at onset $(\mathrm{p}=0.03)$ and a 3.9 decrease with each increase in category of lead time duration $(\mathrm{p}=0.014)$. Outcome was significantly benefitted by steroid therapy in children whose spasms had no identifiable etiology $(\mathrm{p}=0.004)$. Prompt diagnosis and prompt treatment of infantile spasms may help prevent subsequent developmental delay. Younger infants may be more at risk from epileptic encephalopathy than older infants. (O'Callaghan FJK, Lux AL, Darke K, et al. The effect of lead time to treatment and of age of onset on developmental outcome at 4 years in infantile spasms: Evidence from the United Kingdom Infantile Spasms Study. Epilepsia July 2011;52(7):1359-1364). (Response: Professor John P Osborne, Children's Centre, Royal United Hospital, Combe Park, Bath BA1 3NG, UK. E-mail:mpsjpo@bath.ac.uk).

COMMENT. The UKISS was a clinical trial comparing the effects of hormonal treatments (prednisolone or tetracosactide depot) to vigabatrin. Hormonal treatment was superior to vigabatrin in control of spasms, regardless of etiology (Lux et al, 2004), and developmental VABS scores at 14 months and 4 years of age were better in patients receiving hormonal treatment and spasms with no identifiable etiology (Darke et al, 2010).

Early control of spasms and a shorter interval between onset and time of treatment reduce the duration of epileptic encephalopathy, resulting in benefit to developmental outcome. Duration of hypsarrhythmia longer than 3 weeks is also shown to affect developmental outcome (Rener-Primec Z et al, 2006).

\section{OCCIPITAL CORTEX INITIATING GENERALIZED EPILEPSY IN JEAVONS SYNDROME}

Researchers at the Hospital for Sick Children, Toronto, studied the interictal, ictal, and clinical findings on video-EEG in 12 children (11 female; mean age 4.9 years, range 1.5 - 9 years) with Jeavons syndrome (JS). All met the diagnostic criteria of JS: eyelid myoclonia, eye closure-induced seizures/EEG paroxysms, and photosensitivity. Six $(50 \%)$ had a previous diagnosis of absence seizures and 10 were taking antiepileptic medications. All 12 had normal posterior dominant alpha rhythm, reactive to eye opening and closure. Six had spiky posterior alpha activity with sustained eye closure, $10(83 \%)$ had generalized epileptiform discharges (ED) interictally, 11 (92\%) had focal posterior ictal EDs, and 9 (75\%) had eyelid myoclonia and/or paroxysmal EDs induced by photic 
stimulation and by hyperventilation in 7 (58\%). JS patients show focal posterior interictal EDs and focal posterior ictal EDs preceding generalized EDs. The findings suggest that seizures and EEG paroxysms induced by eye closure, intermittent photic stimulation, and hyperventilation are indicative of the occipital cortex initiating the generalized epilepsy network in JS. (Viravan S, Go C, Ochi A, Akiyama T, Snead OC III, Otsubo H. Jeavons syndrome existing as occipital cortex initiating generalized epilepsy. Epilepsia July 2011;52(7):1273-1279). (Respond: Hiroshi Otsubo MD, Division of Neurology, The Hospital for Sick Children, 555 University Avenue, Toronto, ON M5G 1X8, Canada. Email: hiroshi.otsubo@sickkids.ca).

COMMENT. Jeavons syndrome (JS) is referred to as an idiopathic "eye closureinduced" reflex idiopathic generalized epilepsy. In the 2006 ILAF classification of epileptic syndromes, JS was not included as an epileptic syndrome, but eyelid myoclonia was classified as a generalized seizure. Panayiotopoulos (2005) proposed the inclusion of JS as a reflex epilepsy, and Striano et al (2009) as an idiopathic generalized epilepsy. Under the revised terminology (2005-2009) for the ILAE, proposed by Berg AT et al (Epilepsia 2010;51(4):676-685), JS would probably be included as an electroclinical syndrome and a distinctive "constellation" of "genetic" or "unknown" etiology. Four (33\%) of the patients in the above series had a family history of epilepsy.

\section{PREDICTORS OF SEIZURE FREEDOM AFTER SURGERY FOR CORTICAL MALFORMATIONS}

Researchers at the Northern California Epilepsy Center and University of California, San Francisco, conducted a retrospective review of 143 patients with malformations of cortical development (MCD) who underwent resection surgery for medically refractory epilepsy. Median age of seizure onset was 7 years (range, 0-55 years) with $123(86 \%)$ having childhood onset $(<18$ years $)$. Complex partial seizures were most common (47.5\%) followed by generalized tonic-clonic (23\%). Preoperative MRI, classified according to a new MCD classification, included subcortical T2/FLAIR abnormality $(46.1 \%)$, blurring of gray-white junction (26.6\%), cortical thickening $(17.2 \%)$, and mesiotemporal sclerosis (16.1\%). ECoG revealed epileptiform activity in 111 patients, focal in $67.7 \%$. Total resection of abnormal area was achieved in $68.1 \%$. Heterotopia were present in $35 \%$, and neuronal dysplasias in $33.3 \%$. According to the Barkovich classification for MCD, 22\% were class I (abnormal neuronal and glial proliferation), 10\% class II (abnormal migration), 65\% class III (abnormal organization), and 3\% class IV (abnormal development). Class I patients had better outcome than class II or III. Complete resection of ECoG and anatomic abnormalities was the main independent predictor of seizure freedom. Other favorable predictors were smaller lesions and locally confined ECoG abnormalities. Long-term follow-up demonstrated seizure control in $72 \%$ at 2 years, $65 \%$ at 5 years, and $67 \%$ at 10 years. (Chang EF, Wang DD, Barkovich AJ et al. Predictors of seizure freedom after surgery for malformations of cortical development. Ann Neurol July 2011;70(1):151-162). (Respond: Dr Chang, Department of Neurological Surgery, University of California, San Francisco, 505

Parnassus Avenue, M779, San Francisco, CA 94143. E-mail: changed@neurosurg.ucaf.edu). 